\title{
Focusing, Defocusing, and Refraction in a Circularly Stratified Atmosphere ${ }^{1}$
}

\author{
K. Toman ${ }^{2}$
}

(October 29, 1959)

\begin{abstract}
Focusing, defocusing, astronomical refraction and path length of rays as a function of the departure angle $\Delta$ of the ray at the source is described for cases with the source outside, inside, or on the boundary of a circular stratification. Relative to zero elevation angle symmetrical and centrosymmetrical distributions are found.
\end{abstract}

\section{Introduction}

In order to determine focusing, refraction, and path-length properties of rays in a circularly stratified atmosphere for all departure angles at a source, the earth is temporarily removed. If the earth is restored the use of the obtained distributions is unaffected for positive departure angles, but the use of negative departure angles is limited by the elevation of the source. Applied to a spherical lense system, however, the distributions apply for all departure angles.

\section{Focusing and Defocusing}

Place an electromagnetic emitter $(P$ at $r=C \neq 0)$ in a medium of constant refractive index $\left(\mu_{1}\right)$ which is circularly bounded. Designate $\mu_{1}\left(\leq r_{1}\right)=1$, $\mu_{2}\left(r_{1}<r<r_{2}\right)<1$, and $\mu_{3}\left(\geq r_{2}\right)=1$. Define zero elevation angle as the direction $s$ which is normal to the diameter through $P$. Let the smaller quadrant contain positive elevation angles. Assume an opening angle $\delta \Delta$ and let the beam rotate on $P$. The behavior of the opening angle of the refracted beam shows that for positive elevation angles the beam is defocused. For negative elevation angles the beam is focused. If $\mu_{2}\left(r_{1}<r<r_{2}\right)>1$ these conditions are reversed. If the beam is centered in a direction $\Delta=0$ there is neither focusing nor defocusing. Geometrically, this is true for any beam width. For rays the beam width is limited by the known restrictions of ray theory.

These results apply to a circularly stratified ionosphere without magnetic field for which $\mu_{2}(r)<1$ if the influence of the collisional frequency is neglected. Taking into account collisional frequency, the refractive index can become greater than unity and the net effect is either focusing or defocusing. If, however, defocusing is obtained for a particular value of $\Delta$ defocusing strictly prevails over the full quadrant

1 Presented, in parts, at the spring URSI meeting, May 5 to 7, 1959, Washington, D.C.

2 Geophysics Research Directorate, Ionospheric Physies Laboratory, Air Force Cambridge Research Center, Bedford, Mass. (invited paper). to which $\Delta$ belongs. Here, rays are assumed to originate at $P$. Should the source be at the location of a circularly orbiting satellite monitored from $P$ the reciprocity theorem can be invoked.

To illustrate these effects, the differential refraction angle $\delta \epsilon$ of a beam with a beam width $\delta \Delta=5^{\circ}$ passing through a medium $\mu_{1}=\mu_{3}=1, \mu_{2}=0.92$ is shown in figure 1 . The $\delta \epsilon(\Delta)$ distribution was obtained using convenient values for the radial distance of $P$ at $C=90$ with $r_{1}=100$ and $r_{2}=110$. Before entering the refracting medium rays are linear. At $\Delta=90^{\circ}$ (zenith angle $z=0$ ) there remains a net amount of defocusing which is zero for zero beam width. For smaller values of $\Delta$ defocusing is enhanced until a maximum is reached. For $\Delta=0$ there is neither focusing nor defocusing. The $\delta \epsilon(\Delta)$ distribution is centrosymmetrical relative to $\Delta=0$. Similar distributions are obtained if $\mu_{2}$ varies with

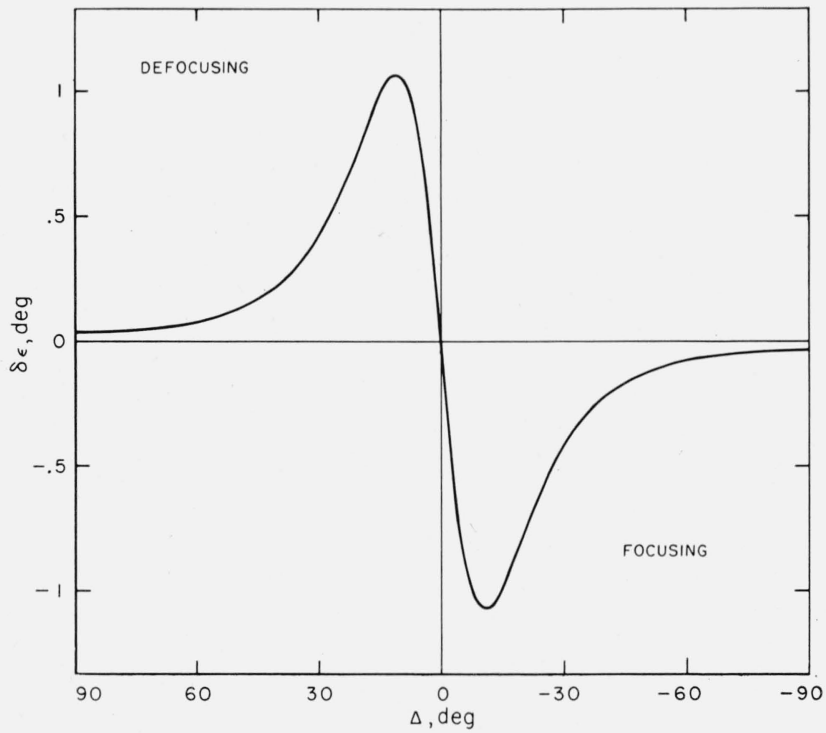

Figure 1. Focusing and defocusing for a circularly stratified medium. 
height in an arbitrary manner. The $\delta \epsilon(\Delta)$ distribution remains centrosymmetrical if $P$ lies at the boundary of stratification provided at this boundary $\mu$ is single valued. If $P$ lies inside the stratification between $r_{1}$ and $r_{2}$, the $\delta \epsilon(\Delta)$ distribution no longer retains its centrosymmetry because for negative departure angles at $P$ the amount of differential refraction depends on $\mu(r)$ for $r<C$.

\section{Refraction}

For the same parameters of figure 1 the astronomical refraction $\delta \eta$ was obtained as shown in figure 2 . Maximum deviation of the ray from its initial direction at $P$ occurs at $\Delta=0$. An interesting case is $\delta \eta=0$ for $\Delta \neq 90^{\circ}$. Then $\delta \eta$ is zero for all values of $\Delta$. Consequently, $\delta \epsilon$ is zero and zero-focusing prevails for all departure angles at $P$.

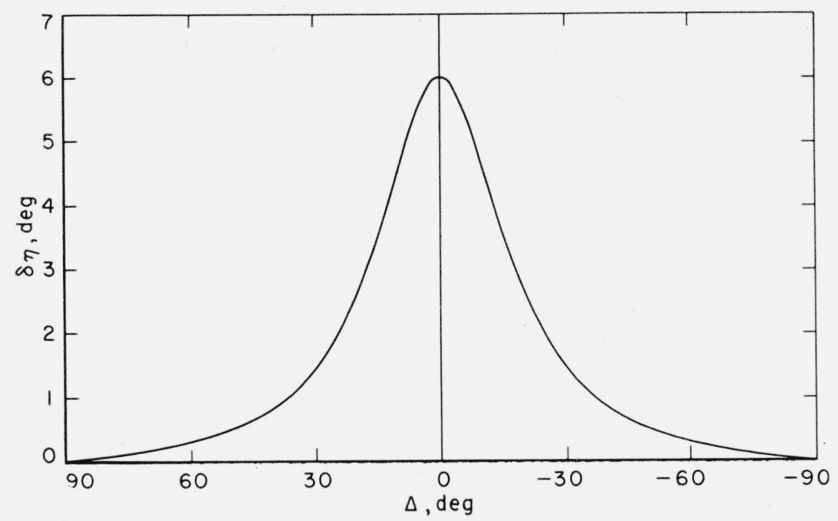

Figure 2. Astronomical refraction $\delta \eta$ for a circularly stratified medium.

The symmetry of $\delta \eta$ is valid for a circularly stratified atmosphere of any $\mu(r)$ distribution provided the lowest stratification level is at $r_{1} \geq C$ and $\mu$ is single valued at $r_{1}=C$. For $r_{1}=C$ the $\delta \eta(\Delta)$ distribution will no longer have zero gradient at $\Delta=0$ since the behavior of $\delta \eta$ depends on the gradient of $\mu$ at the departure point $P$. If $\mu$ at $r_{1}=C$ is double valued the $\delta \eta(\Delta)$ distribution ceases to be symmetrical. For reasons indicated above the $\delta \eta(\Delta)$ distribution also ceases to be symmetrical if $P$ lies inside the stratification.

To make use of the symmetry a radio source must be located there where $\mu$ does not change with height. These conditions may best be fulfilled in the space between the troposphere and the ionosphere. At such a location one obtains extremes of $\delta \epsilon(\Delta)$ and $\delta \eta(\Delta)$ at $\Delta=0$. These distributions are limited by a negative elevation angle for which the ray grazes the "top" of the troposphere.

Snell's law for spherical refracting surfaces derives the orientation of the ray from the boundary values of the $\mu(r)$ distribution. For a circularly stratified atmosphere it is thus possible to determine the $\delta \eta(\Delta)$ distribution for any $\mu(r)$ distribution in a simple manner. First, one chooses a departure angle $\Delta \neq 90^{\circ}$ and traces the ray path from $r_{1}$ to $r_{2}$. Between $r_{1}$ and $r_{2}$ one selects $\mu=$ constant such that the linear ray intersects the actual ray path at $r_{1}$ and $r_{2}$. Outside $r_{2}, \mu$ is chosen so that the angle of intersection of the ray with the radius vector is the same. For this model the complete $\delta \eta(\Delta)$ distribution is easily computed.

\section{Ray-Path Length}

Symmetry properties with respect to zero elevation angle $\Delta$ can also be stated in a general form for the length of ray paths which originate at $P$ and which traverse a circularly stratified medium. If $P$ lies below the boundary of stratification the path length between $r_{1}$ and $r_{2}$ of the refracted ray is a maximum for zero departure angle of the ray at $P{ }^{3}{ }^{3}$ The path-length distribution is symmetrical relative to $\Delta=0$ at $P$. If $P$ lies on the boundary of stratification $\left(C=r_{1}\right)$ this symmetry is preserved provided $\mu$ at $C$ is single valued. If $P$ lies inside the stratification the symmetry of the total path length vanishes but the line integrals between fixed levels for the geometrical path length

$$
\int_{r \geq C}^{C<r \leq \infty} d s
$$

and for the optical path length

$$
\int_{r \geq C}^{C<r \leq \infty} \mu d s
$$

of rays emerging from a source have both a maximum for zero departure angle of the ray at $P$. Their values are symmetrical relative to $\Delta=0$.

Applied to a circularly stratified ionosphere for $C \leq r_{1}$, these results illustrate that the maximum of a nondeviative type of absorption is strictly obtained for zero departure angle of the ray at the source.

\section{Conclusion}

If a source of rays $(P$ at $r=C$ ) lies below or on the boundary of a circular stratification of the refractive index, the astronomical refraction and the total ray path length are both symmetrical relative to $\Delta=0$. with a maximum at $\Delta=0$; the differential refraction is centrosymmetrical. If $P$ lies inside the stratification, these symmetries vanish although the line integrals of the geometrical and the optical path length between levels of $r>C$ are symmetrical and obtain a maximum at $\Delta=0$.

${ }^{3} \mathrm{~K}$. Toman, New geometrical properties and their usefulness for ionospheric: radio propagation, Proc. IRE 47, 1381 (1959).

Boulder, Colo.

(Paper 64D3-592 